\title{
ENDPOINT ESTIMATES FOR THE MAXIMAL OPERATOR ASSOCIATED TO SPHERICAL PARTIAL SUMS ON RADIAL FUNCTIONS
}

\author{
ELENA ROMERA AND FERNANDO SORIA
}

(Communicated by J. Marshall Ash)

\begin{abstract}
Let $T f(x)=\sup _{R>0}\left|S_{R} f(x)\right|$ where $S_{R}$ is the spherical partial sum operator. We show that $T$ is bounded from the Lorentz space $L_{p_{i}, 1}\left(\mathbf{R}^{n}\right)$ into $L_{p_{i}, \infty}\left(\mathbf{R}^{n}\right), i=0,1$ when acting on radial functions and where $p_{0}=$ $\frac{2 n}{n+1}, p_{1}=\frac{2 n}{n-1}$.
\end{abstract}

\section{INTRODUCTION}

Consider the multiplier operator defined by:

$$
(S f)^{\wedge}(\xi)=\chi_{B(0,1)}(\xi) \hat{f}(\xi)
$$

where $B(0,1)$ represents the ball of centre 0 and radius 1 , and ' stands for the Fourier Transform.

In 1954 , Herz [H] showed that $S$ is bounded on $L_{\mathrm{rad}}^{p}\left(\mathbf{R}^{n}\right) \equiv L^{p}\left(\mathbf{R}^{n}\right) \cap$ \{radial functions if and only if $\frac{2 n}{n+1}<p<\frac{2 n}{n-1}$.

A study of $S$ over general functions of $L^{p}\left(\mathbf{R}^{n}\right)$ was made by C. Fefferman who in his celebrated article [F] proved that $S$ is bounded on $L^{p}\left(\mathbf{R}^{n}\right)$ if and only if $p=2$.

Later on, Kenig and Tomas [KT] showed that $S$ is not weak-type on $L_{\mathrm{rad}}^{p}\left(\mathbf{R}^{n}\right)$ for $p=\frac{2 n}{n+1}$, but Chanillo [Ch] obtained a positive result showing that $S$ is of restricted weak-type on $L_{\mathrm{rad}}^{p}\left(\mathbf{R}^{n}\right)$ for the same $p$.

More recently, A. Córdoba [C] and G. Mockenhaupt [M] have proved that $S$ is bounded on the mixed normed space $L_{\mathrm{rad}}^{p} L_{\text {ang }}^{2}\left(\mathbf{R}^{n}\right)$ for the same range of $p, \frac{2 n}{n+1}<p<\frac{2 n}{n-1}$.

Let us define the partial sums $S_{R}$ by

$$
S_{R} f(x)=S f^{R}(R x)
$$

where $f^{R}(x)=f\left(\frac{x}{R}\right)$, and

$$
T f(x)=\sup _{R>0}\left|S_{R} f(x)\right| .
$$

Received by the editors August 9, 1989.

1980 Mathematics Subject Classification (1985 Revision). Primary 42B10, 42B25. 
The aim of this article is to prove the following endpoint estimate:

Theorem 1. $T$ is bounded from $L_{\mathrm{rad}}^{p_{i}, 1}\left(\mathbf{R}^{n}\right)$ into $L_{\mathrm{rad}}^{p_{i}, \infty}\left(\mathbf{R}^{n}\right), i=0,1$ when $p_{0}=$ $\frac{2 n}{n+1}$ and $p_{1}=\frac{2 n}{n-1}$.

This operator has recently been studied by $\mathrm{E}$. Prestini $[\mathrm{P}]$, and independently by Y. Kanjin [K], who proved that $T$ is bounded on $L_{\mathrm{rad}}^{p}\left(\mathbf{R}^{n}\right)$ when $\frac{2 n}{n+1}<p<$ $\frac{2 n}{n-1}$.

Observe that since $T$ maps radial functions into radial functions, we can view $T$ as an operator acting on functions defined on the interval $(0, \infty)$, and then Theorem 1 says that $T$ maps $L^{p_{i}, 1}((0, \infty), d \nu)$ into $L^{p_{i}, \infty}((0, \infty), d \nu)$ where $d \nu$ is the measure $r^{n-1} d r$ and $i=0,1$. Thus, by using Stein-Weiss' extension of the Marzinkiewicz Interpolation Theorem (see [SW]) we obtain as a corollary Prestini and Kanjin's result that we now state separately

Corollary 2. $T$ maps $L_{\mathrm{rad}}^{p}\left(\mathbf{R}^{n}\right) \longrightarrow L_{\mathrm{rad}}^{p}\left(\mathbf{R}^{n}\right)$ for $\frac{2 n}{n+1}<p<\frac{2 n}{n-1}$.

Also, by the usual arguments we obtain the convergence result as a consequence of Theorem 1 ,

Corollary 3. Let $f \in L_{\text {rad }}^{p_{i}, 1}\left(\mathbf{R}^{n}\right), i=0,1$. Then, $\lim _{R \rightarrow \infty} S_{R} f(x)=f(x)$ a.e.

\section{TeChNical Lemmas}

For the proof of Theorem 1 we will need two lemmas about some known operators, the Hardy-Littlewood maximal operator, $M$; the maximal Hilbert transform, $\widetilde{H}$; the transposed of the Hardy operator, $H_{a}^{t}$; and Carleson's operator, $\widetilde{C}$, defined respectively by:

$$
\begin{aligned}
& M g(x)=\sup _{h} \frac{1}{2 h} \int_{x-h}^{x+h}|g(t)| d t, \\
& \tilde{H} g(x)=\sup _{N>\varepsilon>0}\left|\int_{\varepsilon<|t|<N} \frac{g(x-t)}{t} d t\right|, \\
& H_{a}^{t} g(x)=\int_{x}^{\infty}|g(t)| \frac{d t}{t}, \\
& \widetilde{C} g(x)=\sup _{r>0, n \in \mathbf{R}}\left|\int_{-r<x-t<r} \frac{e^{-i n t} g(t)}{x-t} d t\right| .
\end{aligned}
$$

Observe that $H_{a}^{t}$ has been modified by considering first the absolute value of the function. This does not affect its boundedness properties.

Lemma 1. Let $f$ be a radial function and let $f_{0}$ denote its radial projection; i.e. $f(x)=f_{0}(|x|)$. Define the operator $\mathscr{M} g(t)=\left(M+\widetilde{H}+H_{a}^{t}+\widetilde{C}\right)(g)(t)$, then:

$$
T f(x) \leq \frac{c_{n}}{|x|^{\frac{n-1}{2}}} \mathscr{M}\left(f_{0}(r) r^{\frac{n-1}{2}}\right)(|x|) .
$$


This is the crucial estimate in E. Prestini's work, and from it Corollary 2 follows directly. For the sake of completeness we present below a straightforward proof of this inequality. The other lemma concerning these operators is the following weighted inequality:

Lemma 2. With the same notation, we have for every $1<p<\infty$ and every $w \in A_{p}$

$$
\int_{-\infty}^{\infty}|\mathscr{M} g(t)|^{p} w(t) d t \leq c_{p} \int_{-\infty}^{\infty}|g(t)|^{p} w(t) d t
$$

We will also need some inequalities for the Bessel functions $J_{k}(t)$,

Lemma 3. Let $k$ be half of an integer and set

$$
L_{k}(t)=J_{k}(t)-\frac{\sqrt{2 / \pi}}{t^{1 / 2}} \cos \left(t-(2 k-1) \frac{\pi}{4}\right)
$$

and

$$
R_{k}(t)=J_{k}^{\prime}(t)+\frac{\sqrt{2 / \pi}}{t^{1 / 2}} \sin \left(t-(2 k-1) \frac{\pi}{4}\right) .
$$

Then, there exists a finite constant $c_{k}>0$ such that $\forall t>0$ :

(i) $\left|J_{k}(t)\right|,\left|J_{k}^{\prime}(t)\right| \leq c_{k}$,

(ii) $\left|t^{1 / 2} J_{k}(t)\right|,\left|t^{1 / 2} J_{k}^{\prime}(t)\right| \leq c_{k}$,

(iii) $\left|t^{1 / 2} L_{k}(t)\right|,\left|t^{1 / 2} R_{k}(t)\right| \leq c_{k}$,

(iv) $\left|t^{3 / 2} L_{k}^{\prime}(t)\right|, \mid t^{3 / 2} R_{k}^{\prime}(t) \leq c_{k}$.

Proof of Lemma 3. The following recurrence formulae which can be found in [W] p. 45,

$$
\left(t^{k} J_{k}(t)\right)^{\prime}=t^{k} J_{k-1}(t), \quad\left(t^{-k} J_{k}(t)\right)^{\prime}=-t^{-k} J_{k+1}(t)
$$

give rise to the relation:

$$
J_{k}^{\prime}(t)=\frac{1}{2}\left(J_{k-1}(t)-J_{k+1}(t)\right)
$$

which translates properties of $J_{k}$ into properties of $J_{k}^{\prime}$.

Now, estimates (i) and (ii) are well known and (iii) follows inmediately from (ii).

Also, we know (see [SW] p. 158) that there is a $c_{k}$ such that $\left|t^{3 / 2} L_{k}(t)\right| \leq c_{k}$ as $t \longrightarrow \infty$. This inequality is clearly also true for $t \longrightarrow 0$, from definition and part (i). We shall deduce (iv) from this estimate.

Let

$$
V_{k}(t)=\frac{\sqrt{2 / \pi}}{t^{1 / 2}} \cos \left(t-(2 k-1) \frac{\pi}{4}\right)
$$

then

$$
V_{k}^{\prime}(t)=-\frac{\sqrt{2 / \pi}}{2 t^{3 / 2}} \cos \left(t-(2 k-1) \frac{\pi}{4}\right)-\frac{1}{2}\left(V_{k-1}(t)-V_{k+1}(t)\right)
$$


Using the expression of $J_{k}^{\prime}$ in terms of $J_{k-1}$ and $J_{k+1}$ we obtain:

$$
\begin{aligned}
L_{k}^{\prime}(t) & =J_{k}^{\prime}(t)-V_{k}^{\prime}(t) \\
& =\frac{1}{2}\left(L_{k-1}(t)-L_{k+1}(t)\right)+\frac{\sqrt{2 / \pi}}{2 t^{3 / 2}} \cos \left(t-(2 k-1) \frac{\pi}{4}\right) .
\end{aligned}
$$

Thus, the first part of (iv) is proved.

For the second part observe that

$$
\begin{aligned}
R_{k}(t) & =J_{k}^{\prime}(t)-\frac{1}{2}\left(V_{k-1}(t)-V_{k+1}(t)\right) \\
& =\frac{1}{2}\left(L_{k-1}(t)-L_{k+1}(t)\right) .
\end{aligned}
$$

So,

$$
R_{k}^{\prime}(t)=\frac{1}{2}\left(L_{k-1}^{\prime}(t)-L_{k+1}^{\prime}(t)\right)
$$

and we obtain the bound for $R_{k}^{\prime}$ directly from the previous one.

Proof of Lemma 1. Let $f$ be radial, then $\hat{f}$ is also radial and:

$$
\hat{f}(\xi)=2 \pi \int_{0}^{\infty} \frac{J_{\frac{n-2}{2}}(2 \pi t|\xi|) f_{0}(t)}{(t|\xi|)^{\frac{n-2}{2}}} t^{n-1} d t .
$$

So, we have

$$
\begin{aligned}
S_{R} f(r) & =\frac{c}{r^{\frac{n-2}{2}}} \int_{0}^{\infty} s^{n / 2} f_{0}(s) \int_{0}^{R} J_{\frac{n-2}{2}}(2 \pi t s) J_{\frac{n-2}{2}}(2 \pi t r) t d t d s \\
& =\frac{c}{r^{\frac{n-1}{2}}} \int_{0}^{\infty} s^{\frac{n-1}{2}} f_{0}(s) K_{R}(r, s) d s .
\end{aligned}
$$

Where we have defined

$$
K_{R}(r, s)=\sqrt{r s} \int_{0}^{R} J_{\frac{n-2}{2}}(2 \pi t s) J_{\frac{n-2}{2}}(2 \pi t r) t d t=R K_{1}(R r, R s) .
$$

The proof will be finished if we can show that the inequality in Lemma 1 holds for $S_{R}$ independently of $R$.

Reduction. Observe that we would obtain the bound for every $R$ if we could show it just for $R=1$, because all the operators appearing in $\mathscr{M}$ are invariant under the dilations given by $g^{R}(x)=g\left(\frac{x}{R}\right)$. That is, $\mathscr{M}\left(g^{R}\right)(R t)=\mathscr{M} g(t)$.

Bound. We prove now the bound for $\left|S_{1} f\right|$.

$$
S_{1} f(r)=\frac{c}{r^{\frac{n-1}{2}}} \int_{0}^{\infty} s^{\frac{n-1}{2}} f_{0}(s) K_{1}(r, s) d s .
$$

To simplify notation let $g(s)=s^{\frac{n-1}{2}} f_{0}(s)$ and also write $J_{k}(t)$ instead of $J_{k}(2 \pi t)$. This last simplification introduces a constant factor $c$.

We can decompose $K_{1}$ as in [C] using the differential equation satisfied by the Bessel functions

$$
x^{2} J_{k}^{\prime \prime}(x)+x J_{k}^{\prime}(x)+\left(x^{2}-k^{2}\right) J_{k}(x)=0
$$


and obtain

$$
\begin{aligned}
K_{1}(r, s) & =\sqrt{r s} \int_{0}^{1} J_{\frac{n-2}{2}}(t s) J_{\frac{n-2}{2}}(t r) t d t \\
& =\sqrt{r s} \frac{r J_{\frac{n-2}{2}}^{\prime}(r) J_{\frac{n-2}{2}}(s)-s J_{\frac{n-2}{2}}(r) J_{\frac{n-2}{2}}^{\prime}(s)}{r^{2}-s^{2}} \\
& =\frac{\sqrt{r s}}{2} \frac{J_{\frac{n-2}{2}}^{\prime}(r) J_{\frac{n-2}{2}}(s)}{r-s}-\frac{\sqrt{r s}}{2} \frac{J_{\frac{n-2}{2}}(r) J_{\frac{n-2}{2}}^{\prime}(s)}{r-s} \\
& +\frac{\sqrt{r s}}{2} \frac{J_{\frac{n-2}{2}}^{\prime}(r) J_{\frac{n-2}{2}}(s)}{r+s}+\frac{\sqrt{r s}}{2} \frac{J_{\frac{n-2}{2}}(r) J_{\frac{n-2}{2}}^{\prime}(s)}{r+s}
\end{aligned}
$$

The terms corresponding to the last two kernels are easy to handle. For instance, for the third one we have,

$$
\begin{aligned}
\mid \int_{0}^{\infty} & g(s) \frac{\sqrt{r s} J_{\frac{n-2}{2}}^{\prime}(r) J_{\frac{n-2}{2}}(s)}{s+r} d s \mid \\
& \leq c_{n} \int_{0}^{\infty} \frac{|g(s)|}{s+r} d s \leq c_{n}\left(\int_{0}^{r} \frac{|g(s)|}{s+r} d s+\int_{r}^{\infty} \frac{|g(s)|}{s+r} d s\right) \\
& \leq \frac{c_{n}}{r} \int_{0}^{r}|g(s)| d s+c_{n} \int_{r}^{\infty} \frac{|g(s)|}{s} d s \\
& \leq c_{n}\left(M+H_{a}^{t}\right)(g)(r) .
\end{aligned}
$$

And similarly, for the fourth one.

For the first term decompose the domain of integration as

$$
\left|\int_{0}^{\infty} g(s) \frac{\sqrt{r s} J_{\frac{n-2}{2}}^{\prime}(r) J_{\frac{n-2}{2}}(s)}{r-s} d s\right|=\left|\int_{0}^{r / 2}\right|+\left|\int_{r / 2}^{3 r / 2}\right|+\left|\int_{3 r / 2}^{\infty}\right|=I+I I+I I I
$$

and estimate the three integrals separately. Clearly,

$$
I \leq c_{n} \int_{0}^{r / 2} \frac{|g(s)|}{r-s} d s \leq \frac{c_{n}}{r} \int_{0}^{r}|g(s)| d s \leq c_{n} M(g)(r)
$$

and

$$
I I I \leq c_{n} \int_{3 r / 2}^{\infty} \frac{|g(s)|}{s} d s \leq c_{n} H_{a}^{t}(g)(r) .
$$

There is some more work to do with $I I$. Using Lemma 3 again we get

$$
\begin{aligned}
I I & \leq c_{n}\left|\int_{r / 2}^{3 r / 2} \frac{s^{1 / 2} \frac{J_{n-2}}{2}(s) g(s)}{r-s} d s\right| \\
& =c_{n}\left|\int_{r / 2}^{3 r / 2} \frac{g(s) s^{1 / 2}\left(J_{\frac{n-2}{2}}(s)-\frac{\sqrt{2 / \pi}}{s^{1 / 2}} \cos \left(s-(n-4) \frac{\pi}{2}\right)+\frac{\sqrt{2 / \pi}}{s^{1 / 2}} \cos \left(s-(n-4) \frac{\pi}{2}\right)\right)}{r-s} d s\right| \\
& \leq c_{n}\left|\int_{r / 2}^{3 r / 2} \frac{g(s) \cos \left(s-(n-4) \frac{\pi}{2}\right)}{r-s} d s\right|+c_{n}\left|\int_{r / 2}^{3 r / 2} \frac{g(s) s^{1 / 2} L_{\frac{n-2}{2}}(s)}{r-s} d s\right|=A+B .
\end{aligned}
$$


$A$ is bounded directly by Carleson's operator $A \leq c_{n} \widetilde{C}(g)(r)$. To estimate $B$, observe that by the Mean Value Theorem there is an $\bar{r} \in\left(\frac{r}{2}, \frac{3 r}{2}\right)$ such that

$$
\begin{aligned}
& B \leq c_{n}\left|L_{\frac{n-2}{2}}(r)\right|\left|\int_{r / 2}^{3 r / 2} \frac{g(s) s^{1 / 2}}{r-s} d s\right|+c_{n} \int_{r / 2}^{3 r / 2} \frac{\left|g(s) s^{1 / 2}\right|}{|r-s|}|r-s|\left|L_{\frac{n-2}{2}}^{\prime}(\bar{r})\right| d s \\
& \leq c_{n}\left|L_{\frac{n-2}{2}}(r)\right|\left|\int_{r / 2}^{3 r / 2} \frac{g(s)\left(s^{1 / 2}-r^{1 / 2}+r^{1 / 2}\right)}{r-s} d s\right|+c_{n} \int_{r / 2}^{3 r / 2}|g(s)| s^{1 / 2}\left|L_{\frac{n-2}{2}}^{\prime}(\bar{r})\right| d s .
\end{aligned}
$$

Now, using part (iv) of Lemma 3:

$$
\begin{aligned}
\leq & c_{n}\left|L_{\frac{n-2}{2}}(r)\right|\left|\int_{r / 2}^{3 r / 2} \frac{g(s)\left(s^{1 / 2}-r^{1 / 2}\right)}{r-s} d s\right|+c_{n}\left|L_{\frac{n-2}{2}}(r)\right|\left|\int_{r / 2}^{3 r / 2} \frac{g(s) r^{1 / 2}}{r-s} d s\right| \\
& +\frac{c_{n}}{r^{3 / 2}} \int_{r / 2}^{3 r / 2}|g(s)| s^{1 / 2} d s .
\end{aligned}
$$

Now, it is easy to see that if $r, s>0$ then $\left|s^{1 / 2}-r^{1 / 2}\right| \leq|s-r| / r^{1 / 2}$. Apply also part (iii) of Lemma 3 to get

$$
\begin{aligned}
& \leq \frac{c_{n}}{r^{1 / 2}}\left|\int_{r / 2}^{3 r / 2} \frac{g(s)}{r^{1 / 2}} d s\right|+c_{n}\left|\int_{r / 2}^{3 r / 2} \frac{g(s)}{r-s} d s\right|+\frac{c_{n}}{r} \int_{r / 2}^{3 r / 2}|g(s)| d s \\
& \leq \frac{c_{n}}{r} \int_{r / 2}^{3 r / 2}|g(s)| d s+c_{n}\left|\int_{r / 2}^{3 r / 2} \frac{g(s)}{r-s} d s\right|+\frac{c_{n}}{r} \int_{r / 2}^{3 r / 2}|g(s)| d s \\
& \leq c_{n}(M+\widetilde{H})(g)(r) .
\end{aligned}
$$

This ends the estimate of the first term of $K_{1}$. The estimate for the second term follows the same pattern, the only difference is that we introduce the function sine instead of the function cosine (with the same factor and constant). Consequently, we need the bounds for $R_{k}$ of Lemma 3.

\section{Proof of the Main Result}

Now we are able to prove Theorem 1.

Proof of Theorem 1. Let $f$ be a radial function. By abuse of notation we will also use $f$ to denote its radial projection $f_{0}$.

For each $k$ define the intervals $I_{k}=\left[2^{k}, 2^{k+1}\right)$ and decompose the set :

$$
\{x: T f(x)>\lambda\}=\bigcup_{k \in \mathbf{Z}}\left\{x:|x| \in I_{k}, T f(x)>\lambda\right\} .
$$

Decompose further the function $f$ for every $k \in \mathbf{Z}$,

$$
f=f \chi_{i_{k}^{*}}+f \chi_{\left(k_{k}^{*}\right) c}=f_{k}^{1}+f_{k}^{2},
$$

where $I_{k}^{*}=\left(2^{k-1}, 2^{k+2}\right)$. From Lemma 1 we have:

$$
T f_{k}^{1}(x) \leq \frac{c_{n}}{|x|^{\frac{n-1}{2}}} \mathscr{M}\left(f_{k}^{1}(r) r^{\frac{n-1}{2}}\right)(|x|) .
$$


On the other hand, if $r \in I_{k}$ and $s \notin I_{k}^{*}$, then $|r-s| \sim r+s$. Using this and (ii) of Lemma 3 we arrive to

$$
\begin{aligned}
\left|K_{R}(r, s)\right| & =\left|R K_{1}(R r, R s)\right| \\
& =\sqrt{r s}\left|\frac{R r J_{\frac{n-2}{2}}^{\prime}(R r) J_{\frac{n-2}{2}}(R s)-R s J_{\frac{n-2}{2}}(R r) J_{\frac{n-2}{2}}^{\prime}(R s)}{r^{2}-s^{2}}\right| \leq \frac{c_{n}}{r+s} .
\end{aligned}
$$

Therefore, if $|x|=r \in I_{k}$,

Hence,

$$
\left|S_{R} f_{k}^{2}(x)\right| \leq \frac{c}{|x|^{\frac{n-1}{2}}} \int_{0}^{\infty} \frac{s^{\frac{n-1}{2}}\left|f_{k}^{2}(s)\right|}{r+s} d s \leq \frac{c}{|x|^{\frac{n-1}{2}}} \int_{0}^{\infty} \frac{s^{\frac{n-1}{2}}|f(s)|}{|x|+s} d s .
$$

$$
\begin{aligned}
|\{x: T f(x)>\lambda\}| \leq & \left|\left\{x: \frac{1}{|x|^{\frac{n-1}{2}}} \int_{0}^{\infty} \frac{s^{\frac{n-1}{2}}|f(s)|}{|x|+s} d s>c \lambda\right\}\right| \\
& +\sum_{k \in \mathbf{Z}}\left|\left\{x:|x| \in I_{k}, \frac{1}{|x|^{\frac{n-1}{2}}} \mathscr{M}\left(f_{k}^{1}(r) r^{\frac{n-1}{2}}\right)(|x|)>c \lambda\right\}\right| \\
= & I+I I .
\end{aligned}
$$

To estimate $I I$ observe that $|x| \in I_{k}$ implies $\frac{1}{|x|^{\frac{n-1}{2}}}<\frac{1}{2^{k^{\frac{n-1}{2}}}}$ and by Chebychev we have for $1<p<\infty$,

$$
\begin{aligned}
I I & \leq \sum_{k \in \mathbf{Z}} \frac{1}{\lambda^{p} 2^{k p \frac{n-1}{2}}} \int_{I_{k}}\left|\mathscr{M}\left(f_{k}^{1}(r) r^{\frac{n-1}{2}}\right)(s)\right|^{p} s^{n-1} d s \\
& \leq c_{n} \sum_{k \in \mathbf{Z}} \frac{2^{k(n-1)}}{\lambda^{p} 2^{k p \frac{n-1}{2}}} \int_{I_{k}}\left|\mathscr{M}\left(f_{k}^{1}(r) r^{\frac{n-1}{2}}\right)(s)\right|^{p} d s .
\end{aligned}
$$

Using now Lemma 2 with $w(s) \equiv 1$,

$$
\begin{aligned}
& \leq c_{n} \sum_{k \in \mathbf{Z}} \frac{2^{k(n-1)}}{\lambda^{p} 2^{k p \frac{n-1}{2}}} \int_{I_{k}^{*}}\left|f_{k}^{1}(s)\right|^{p} s^{p \frac{n-1}{2}} d s \\
& \leq c_{n}^{\prime} \sum_{k \in \mathbf{Z}} \frac{1}{\lambda^{p} 2^{k p \frac{n-1}{2}}} 2^{(k-2) p \frac{n-1}{2}} \int_{I_{k}^{*}}|f(s)|^{p} s^{n-1} d s \\
& \leq \frac{c_{n}^{\prime \prime}}{\lambda^{p}} \int_{\mathbf{R}}|f(s)|^{p} s^{n-1} d s=\frac{c_{n}^{\prime \prime \prime}}{\lambda^{p}}\|f\|_{p}^{p} \leq \frac{c}{\lambda^{p}}\|f\|_{p, 1}^{p} .
\end{aligned}
$$

To estimate $I$ we make use of the fact that $L^{p_{0}, 1} \subset\left(L^{p_{1}, \infty}\right)^{*}$ and $L^{p_{0}^{\prime}, \infty}=$ $\left(L^{p_{1}, 1}\right)^{*}$ and separate the cases where $p=p_{0}$ or $p=p_{1}$. Let us begin with the estimate for $p_{0}$.

Observe that $\frac{1}{|y|^{\frac{n-1}{2}}} \in L^{p_{1}, \infty}$; thus,

$$
\begin{aligned}
\frac{1}{|x|^{\frac{n-1}{2}}} \int_{0}^{\infty} \frac{|f(s)|}{s+|x|} s^{\frac{n-1}{2}} d s & \leq \frac{1}{|x|^{\frac{n+1}{2}}} \int_{0}^{\infty}|f(s)| s^{-\frac{n-1}{2}} s^{n-1} d s \\
& \simeq \frac{1}{|x|^{\frac{n+1}{2}}} \int_{\mathbf{R}^{n}}|f(y)| \frac{1}{|y|^{\frac{n-1}{2}}} d y \leq \frac{c}{|x|^{\frac{n+1}{2}}}\|f\|_{p_{0}, 1} .
\end{aligned}
$$


For $p_{1}$ observe instead that $\frac{1}{|y|^{\frac{n+1}{2}}} \in L^{p_{0}, \infty}$; then,

$$
\begin{aligned}
\frac{1}{|x|^{\frac{n-1}{2}}} \int_{0}^{\infty} \frac{|f(s)|}{|x|+s} s^{\frac{n-1}{2}} d s & \leq \frac{1}{|x|^{\frac{n-1}{2}}} \int_{0}^{\infty}|f(s)| s^{-\frac{n+1}{2}} s^{n-1} d s \\
& \simeq \frac{1}{|x|^{\frac{n-1}{2}}} \int_{\mathbf{R}^{n}}|f(y)| \frac{1}{|y|^{\frac{n+1}{2}}} d y \leq \frac{c}{|x|^{\frac{n-1}{2}}}\|f\|_{p_{1}, 1} .
\end{aligned}
$$

Combining both estimates we obtain

$$
I=\left|\left\{x: \frac{1}{|x|^{\frac{n-1}{2}}} \int_{0}^{\infty} \frac{|f(s)|}{|x|+s} s^{\frac{n-1}{2}} d s>c \lambda\right\}\right| \leq \frac{c_{n}}{\lambda^{p_{i}}}\|f\|_{p_{i}, 1}^{p_{i}} \quad i=0,1 .
$$

So, the proof of Theorem 1 is finished.

\section{FinAl REMARKS}

(a) Observe that our proof gives actually the following stronger result:

Theorem 1'. $T$ maps $\left(L_{\mathrm{rad}}^{p_{0}, \infty}\right)^{*}$ into $L_{\mathrm{rad}}^{p_{1}, \infty}$ and, analogously, $\left(L_{\mathrm{rad}}^{p_{1}, \infty}\right)^{*}$ into $L_{\mathrm{rad}}^{p_{0}, \infty}$.

(b) During the process of writing this paper we have learned from L. Colzani that A. Crespi has obtained the same estimates as in our Theorem 1. The techniques he uses for the convergence at endpoints follow some ideas that one can find in [Co].

\section{REFERENCES}

[Ch] S. Chanillo, The multiplier for the ball and radial functions, J. Funct. Anal. 55 (1984), 18-24.

[Co] L. Colzani, Convergence of expansions in Legendre polynomials, preprint.

[C] A. Cordoba, The disk multiplier, Duke Math. J. 58 (1989), 21-27.

[F] C. Fefferman, The multiplier for the ball, Ann. of Math. 94 (1971), 330-336.

[H] C. Herz, On the mean inversion of Fourier and Hankel transforms, Proc. Nat. Acad. Sci. U.S.A. 40 (1954), 996-999.

[K] Y. Kanjin, Convergence and divergence almost everywhere of spherical means for radial functions, Proc. Amer. Math. Soc. 103 (1988), 1063-1069.

[KT] C. Kenig and P. Tomas, The weak behaviour of spherical means, Proc. Amer. Math. Soc. 78 (1980), 48-50.

[M] G. Mockenhaupt, On radial weights for the spherical summation operator, J. Funct. Anal. 91 (1990), 174-181.

[P] E. Prestini, Almost everywhere convergence of the spherical partial sums for radial functions, Monatsh. Math. 105 (1988), 207-216.

[SW] E. Stein and G. Weiss, Introduction to Fourier analysis on euclidean spaces, Princeton Univ. Press, 1971.

[W] G. N. Watson, A treatise on the theory of Bessel functions, Cambridge Univ. Press., 1966.

Departamento de Matemáticas, Universidad Autónoma, 28049 Madrid, Spain 